September . 18, $2018 \quad 20: 23$ WSPC/INSTRUCTION FILE

\title{
EMERGENT NETWORK STRUCTURE, EVOLVABLE ROBUSTNESS AND NON-LINEAR EFFECTS OF POINT MUTATIONS IN AN ARTIFICIAL GENOME MODEL
}

\author{
THIMO ROHLF \\ Epigenomics program, Genopole, 523 Terasses de l'Agora, F-91000 Evry, France \\ Max-Planck-Institute for Mathematics in the Sciences, \\ Inselstr. 22, D-04103 Leipzig, Germany \\ rohlf@santafe.edu \\ CHRIS WINKLER \\ Pioneer Hi-Bred International, 7250 NW 62nd Ave., \\ Johnston, IA 50131, USA \\ chris.winkler@pioneer.com
}

\begin{abstract}
Genetic regulation is a key component in development, but a clear understanding of the structure and dynamics of genetic networks is not yet at hand. In this paper we investigate these properties within an artificial genome model originally introduced by Reil [17]. We analyze statistical properties of randomly generated genomes both on the sequenceand network level, and show that this model correctly predicts the frequency of genes in genomes as found in experimental data. Using an evolutionary algorithm based on stabilizing selection for a phenotype, we show that dynamical robustness against single base mutations, as well as against random changes in initial states of regulatory dynamics that mimic stochastic fluctuations in environmental conditions, can emerge in parallel. Point mutations at the sequence level have strongly non-linear effects on network wiring, including as well structurally neutral mutations and simultaneous rewiring of multiple connections, which occasionally lead to strong reorganization of the attractor landscape and metastability of evolutionary dynamics. Evolved genomes exhibit characteristic patterns on both sequence and network level.
\end{abstract}

Keywords: artificial genome,gene regulatory network,evolution

\section{Introduction}

The transcription of DNA into mRNA and subsequent translation into protein is the fundamental genetic process; it is the crucial first step by which genetic information gives rise to an organism. Development is not such a linear process, however. By binding to specific regions of the genome, the protein produced by one gene can affect the activity of other genes, and those genes may in turn express proteins that enhance or inhibit still more genes. A network of interactions responsible for the regulation of genetic activity is thus defined. Such genetic regulation is important if cells are to have independent control over their behavior.

Today, the available amount of data for regulatory interactions in a number of 
model organisms, as, for example, Yeast [21] is steadily increasing. A number of distinguishing structural properties have been identified, namely scale-free degree distributions [11], motifs [6] and modular organization [20].

Still, there is not enough information to suggest a comprehensive theory of how genetic regulatory networks attain a particular structure, how genes in such networks interact and respond to perturbation, and how evolution has shaped these factors. This study is an attempt to explore these questions in the context of one particular model [17], in the hopes that it has features that correspond to the limited data currently available, and so that some progress toward a comprehensive theory might eventually be made.

Traditionally, attempts to understand the characteristics of regulatory networks have focused on dynamical properties. That is, a network topology is specified and rules are applied to describe how each gene in the network responds to inputs. Some initial state is then assigned and the time evolution of gene activity is studied. A variety of rules have been used, including Boolean switches [12], thresholds [14,18], and differential equations [9]. Much less work has been done in understanding how the machinery of transcription, translation, and binding might act throughout the genome to produce the topology of a genetic network. In fact, most studies of genetic networks ignore modeling DNA-specific processes altogether [5]. The first part of our study examines to what extent Reil's model [17], which includes explicit parameterizations for transcription and translation, can produce realistic genetic networks based on random genome realizations.

A description of the method we will use for building genetic regulatory networks follows, along with comparisons to published and publically available experimental data. Statistical properties of random realizations of artificial genomes are derived, and related to network structure. Next, we investigate the dynamics of our modeled networks when applying threshold dynamics to gene behavior. Although this is a strong simplification, this type of discrete dynamics has been successfully applied in a number of studies that are concerned with the co-evolution of network dynamics and -structure [2-4]. Finally, we are interested in understanding the role evolution might play in selecting particular network topologies. This is explored by asking how genome structure changes when those networks with certain dynamical properties are preferentially selected. Similar questions have been addressed in a small number of previous proof-of-principle studies using artificial genomes [1,10,13], however, without relating the observed adaptation to changes in sequence and network topology. In particular, we investigate a scenario of stabilizing selection similar to previous studies concerned with the evolution of developmental canalization [4], and evolution of gene regulatory networks in changing environments [].

We find evolution towards robustness of regulatory dynamics against both noise, modeled as fluctuating initial conditions, and against mutations. We show that, in principle, this phenotypic robustness can be traced back to adaptive changes on the sequence level that lead to emergence of more robust regulatory networks. 


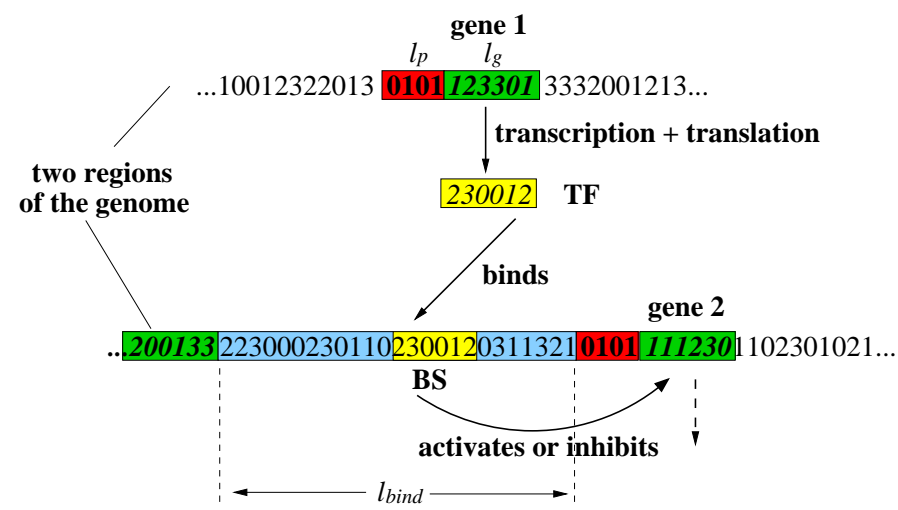

Fig. 1. Schematic description of artificial genome construction (after [17]): a fixed sequence of $l_{p}$ digits - here '0101' (red boxes), $l_{p}=4$ - is defined as promoter sequence. Wherever it occurs on the string defining the genome, the next $l_{g}$ digits are defined as "genes" (here, $l_{g}=6$, green boxes). If the gene is active, a transcription factor (TF) is produced by increasing each digit of the gene sequence by 1 (yellow), crudely mimicking transcription and translation. The algorithm searches matching sequences anywhere in the genome in binding regions (blue box in the lower string) between genes, defining binding sites (BS) for the TF. The so-defined directed regulatory interaction from gene 1 to gene 2 can either activate or inhibit transcription of the next gene downstream (gene 2, dashed arrow). For details, see section 2.1] in the text.

\section{Model Details}

\subsection{Regulatory network construction from random sequences}

An artificial genome can be constructed as follows (also see Fig. 1). Randomly string together $S$ integers drawn uniformly between 0 and 3 . The use of 4 digits need not be the case, but does provide correspondence with the ATGC alphabet of real genomes. For the purpose of generalization, the length of the alphabet in the artificial genome may in principle take any positive integer value $\lambda$. Next, define a base promoter sequence of length $l_{p}$ to indicate the position of genes in the genome, say '0101'. Wherever the promoter sequence occurs, the next $l_{g}$ digits are specified as a gene. Translation of the gene sequence into a protein occurs simply. Each number in the sequence is incremented by 1 and any values greater than the last number in the base set of digits become the first number (e.g., the gene '012323' becomes the protein '123030'). Binding sites are determined by searching the genome for the protein sequence. If a match is found, then the protein is a transcription factor (TF) that binds to that site and that regulates the next downstream gene. In case there are multiple binding sites of this TF for this gene, only one of them is counted for network construction. TFs may enhance or inhibit gene activity. In this study each TF has equal contribution to a gene's state and has equal probability of activating or suppressing gene expression. In real genetic systems, a TF may activate some genes and inhibit others, depending on a complex interplay between various factors that do not only depend on sequence. In our study, we make the simplifying assumption 
that a TF is either activating or inhibiting, which is determined by the sum $s_{g}$ of its sequence: if $s_{g}<(1 / 2) s_{\max }$, where $s_{\max }=(\lambda-1) l_{g}$ is the maximal possible cross sum value, it is inhibiting, otherwise it is activating. Activation and inhibition are reflected by different weight values in the interaction matrix (network) defined by all TFs and their corresponding binding sites (cf. section 2.2).

Clearly this model greatly simplifies the true transcription, translation, and binding processes. The binding of a real transcription factor to a cis-site, for example, depends on the protein's structure, shape, and environment, rather than a simple template matching approach. Moreover, there is a stochastic element to all these processes that is simply ignored here.

Although it represents a strong simplification, the model does have biological justification [17]. The use of a base promoter sequence is reminiscent of the TATA box frequently found in eukaryotic organisms. Binding is modeled in a DNAspecific way, just as in real organisms. Additionally, the model has the potential for greater extendability than some models (e.g., Boolean networks) because it includes DNA-specific transcription, translation, and binding. The impact of single base pair mutations on gene function and network structure can be studied with this model, and also the effect of sequence duplications (resulting in gene duplication) or -deletions [16]. In this paper, we will restrict ourselves to single base pair mutations, and keep the genome size constant, both with respect to the number of bases $S$ and the number of genes $N$.

\subsection{Regulatory dynamics}

Dynamics of state changes (activity or inhibition of genes) on the constructed networks can be defined in various ways. In our study, we apply random threshold network (RTN) dynamics: An RTN consists of $N$ randomly interconnected binary sites (spins) with states $\sigma_{i}= \pm 1$. For each site $i$, its state at time $t+1$ is a function of the inputs it receives from other spins at time $t$ :

$$
\sigma_{i}(t+1)= \begin{cases}+1, & f_{i}(t)>0 \\ -1, & f_{i}(t) \leq 0\end{cases}
$$

with

$$
f_{i}(t)=\sum_{j=1}^{N} c_{i j} \sigma_{j}(t)+h .
$$

The $N$ network sites are updated synchronously. In the following discussion the threshold parameter $h$ is set to zero. The interaction weights $c_{i j}$ take discrete values $c_{i j}=+1$ (activation) or -1 (inhibition); whether a given interaction is activating or inhibiting, is defined by the TF it is derived from, as explained in section 2.1. If $i$ does not receive signals from $j$, one has $c_{i j}=0$.

For a finite system size $N$, the dynamics of RTN, which are closely related to Boolean networks [12] converge to periodic attractors (limit cycles) after a finite 
number of updates. It has been suggested that different limit cycles may correspond to different gene expression states (cell types) [12]. This property of RTN is also advantageous for defining phenotypes in artificial evolutionary scenarios that are subject to various kinds of selective pressure [4].

\section{Statistical properties of the artificial genome}

In the following, $N$ denotes the number of genes in the artificial genome, $S$ the number of bases, $l_{g}$ the length of gene sequences, $l_{p}$ the length of promoter sequences (both are fixed and identical for all genes), and $\lambda$ the length of the alphabet. We now show how these quantities are interrelated via the combinatorial construction of the artificial genome, as outlined in section 2.1 .

\subsection{Statistical distribution of $l_{\text {bind }}$}

Let us first derive the statistical distribution of lengths $l_{\text {bind }}$ of the binding regions preceding promoters in the artificial genome. We incrementally draw a sequence of random digits (bases) from the alphabet. Once we have drawn at least $s \geq l_{p}$ bases, the probability that a promoter sequence is generated by chance with base $s$ is $p_{p}=(1 / \lambda)^{l_{p}}$, since the last $l_{p}$ digits must have position-specific values according to the predefined promoter sequence, and each of these values has probability $1 / \lambda$ to occur. Hence, the probability distribution of the number $X$ of Bernoulli trials (i.e. the sequence length) needed to get one success (a promoter sequence) is a geometric distribution for $s \geq l_{p}$ and zero otherwise,

$$
P(X=s)= \begin{cases}0 & \text { if } 0<s<l_{p} \\ p_{p}\left(1-p_{p}\right)^{s-l_{p}} & \text { if } s \geq l_{p}\end{cases}
$$

Since the last $l_{p}$ digits constitute the promoter, the length of the preceding binding region is given by $l_{\text {bind }}=s-l_{p}$, and it follows

$$
\begin{aligned}
p\left(l_{\text {bind }}\right) & =p_{p}\left(1-p_{p}\right)^{l_{\text {bind }}} \\
& =\lambda^{-l_{p}}\left(1-\lambda^{-l_{p}}\right)^{l_{\text {bind }}} \\
& =\lambda^{-l_{p}} \exp \left[-\alpha \cdot l_{\text {bind }}\right],
\end{aligned}
$$

which is a decaying exponential distribution with $\alpha=-\ln \left(1-\lambda^{-l_{p}}\right)$.

From Eq. 4 follows that the average length of binding regions is given by

$$
\left\langle l_{\text {bind }}\right\rangle=\lambda^{l_{p}}-1
$$

which is the mean of the geometric distribution.

\subsection{Genome size scaling}

From Eq. 3 follows that on average, we have to draw $\lambda^{l_{p}}+l_{p}-1$ bases to obtain a promoter sequence; the next $l_{g}$ bases are defined as the associated gene. Hence, 
to produce genomes with exactly $N$ genes, the expectation value for the number of bases $S$ that we have to string together is

$$
\langle S\rangle=N \cdot\left(\lambda^{l_{p}}+l_{p}-1+l_{g}\right) .
$$

If we instead keep $S$ fixed and ask for the expected number of genes, under the assumption that $l_{p}<l_{g} \ll \lambda^{l_{p}}$, which holds for typical values considered in this study (e.g., $l_{g}=6, l_{p}=4$ and $\lambda=4$ ), we conclude that

$$
\langle N\rangle \approx \frac{1}{\lambda^{l_{p}}} \cdot S
$$

\subsection{Network connectivity}

In this section, we relate the previously derived statistical properties of the artificial genome to characteristic parameters of the resulting random networks.

\subsubsection{Average connectivity}

For a given TF, the probability to match to a random base sequence of length $l_{g}$ is given by $p_{\text {bind }}=\lambda^{-l_{g}}$. There are $n:=\left\langle l_{\text {bind }}\right\rangle-l_{g}+1$ subsequences of length $l_{g}$ in a binding region of expected length $\left\langle l_{\text {bind }}\right\rangle$. The probability that none of these matches the TF sequence is

$$
p_{0}=\left(1-p_{\text {bind }}\right)^{n}
$$

thus the probability that the TF provides at least one input to the gene defined by the promoter sequence following a binding region is a

$$
p_{\text {input }}=1-p_{0}=1-\left(1-\lambda^{-l_{g}}\right)^{\left\langle l_{\text {bind }}\right\rangle-l_{g}+1} .
$$

Since, in a genome with $N$ genes, we have $N$ binding regions and $N$ transcription factors, the total number of regulatory interactions $\left\langle k_{\text {total }}\right\rangle$ per genome (averaged over the whole ensemble of possible random genomes) scales quadratically with the number $N$ of genes,

$$
\left\langle k_{\text {total }}\right\rangle=p_{\text {input }} \cdot N^{2},
$$

and the slope depends on $\lambda, l_{g}$ and $l_{p}$. It follows that the average connectivity (wiring density) $\langle k\rangle:=\left\langle k_{\text {total }}\right\rangle / N$ scales linear with $N$.

Notice, however, that the average number of regulatory interactions $\bar{K}$ obtained from a particular genome realization can substantially deviate from $\langle k\rangle$, since the possible values of $\bar{K}$ are approximately Gaussian distributed [10].

a There is a finite chance that the same TF can bind more than once in a given binding region, however, since the update scheme for network dynamics requires uniquely defined connections, we assign only one regulatory input in this case. If the distance between promoters is smaller than $l_{g}$, no binding occurs, however, for typical parameter values of $\lambda, l_{g}$ and $l_{p}$ as applied in our study, this is a very unlikely event and can be neglected. 


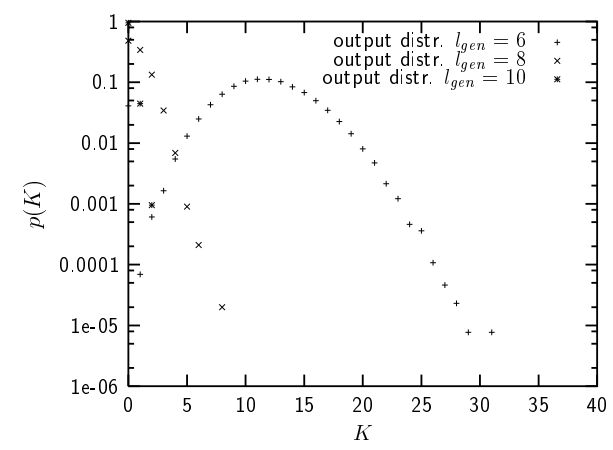

(a) outdegree-distribution

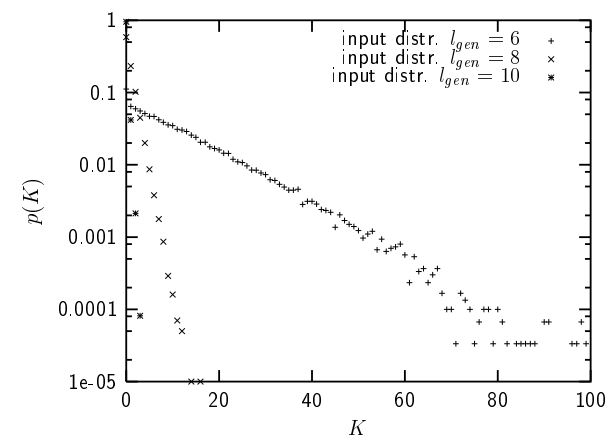

(b) indegree -distribution

Fig. 2. The probability of having $K_{\text {out }}$ regulatory outputs (a) and the probability of having $K_{\text {in }}$ regulatory inputs for random genomes with different gene lengths $l_{g}$, averaged over $10^{4}$ realizations.

\subsubsection{In- and outdegree distribution}

From the above considerations, it is straight-forward to derive the statistical distributions for the number of ingoing and outgoing links in randomly constructed genomes. As denoted in section 2.1 (see also Fig. 1), the subsequent processes of transcription/translation of gene sequences (incrementation of each number in the gene sequence by 1), defining transcription factors $\mathrm{TF}$, and binding of the TF to subsequences of the base string by template matching, defines a network of directed regulatory interactions. A given TF represents an out-link of the gene that codes for it, and an in-link for all other genes that have binding sites for this TF. By definition of template matching, each TF has equal probability $p_{\text {bind }}=\lambda^{-l_{g}}$ to bind at any region of the base string (cf. section 3.3.1), and hence generation of out-links is a Poisson process [7]. Consequently, the outdegree distribution is a Poissonian (Fig. $2 a)$ :

$$
P\left(k_{\text {out }}\right)=\frac{\langle K\rangle^{k_{\text {out }}}}{k_{\text {out }} !} \exp [-\langle K\rangle]
$$

The number of inputs a gene receives from other genes, however, is proportional to the length $l_{\text {bind }}$ of its associated binding region, hence, it follows from Eq. 5

$$
P\left(k_{i n}\right) \sim \exp \left[-\beta k_{i n}\right],
$$

i.e. the indegree distribution is exponential. Both results are confirmed by numerical simulations (Fig. 2a and 2b).

\subsection{Relevance to biology}

Clearly, random genome realizations ar far from being a realistic model of real biological genetic systems. However, it can be shown that even this extreme oversimplification has some relevance for biology. In Figure 3, the predicted number 


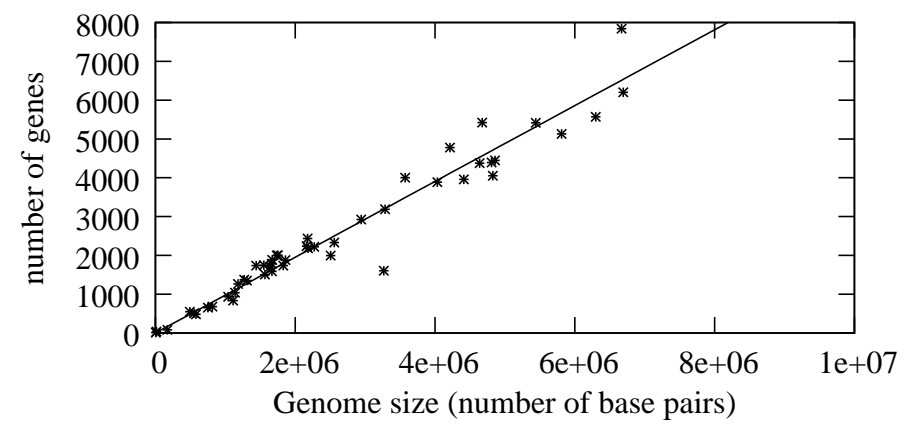

Fig. 3. The number of genes predicted from the model as a function of genome size $S$ with $l_{p}=5$ (line). Data points $(+)$ show the number of genes in 50 prokaryotic organisms, for which complete sequence information is available. Observed data are taken from http://www.ultranet.com/ jkimball/

of genes in a genome, $N=(1 / 4)^{l_{p}} \cdot S$, is plotted as a function of genome size for $l_{p}=5$. Observed data from 50 prokaryotic organisms that have been completely sequenced are also shown. The correspondence between model and data is excellent for this range of $S$ and shows that a combinatorial method for determining the number of genes in a genome is appropriate. For larger $S$, as typically found in eukaryotic organisms, $l_{p}=7$ is reasonable (not shown), but little observed data exists. On the other hand, statistical distributions of regulatory inputs and outputs do not match biological data particularly well; here, more realistic statistics can be obtained by constructing artificial genomes from duplication and divergence events [16]. However, even in these models the question how selection pressure on the phenotype, as encoded by network dynamics, may influence genome organization, remains unanswered. This type of question shall be addressed in the remaining part of this paper.

\section{Stabilizing selection for a phenotype - an evolutionary scenario}

Though evolved by the random processes of genetic drift and selection pressure from changing environments, real genetic systems are far from being random. Complex organization in genome structure is often connected to the highly non-linear nature of the genotype-phenotype map, which includes an intermediate layer of complex regulatory processes controlling cell machinery (unicellular organisms) or highly structured developmental processes (multicellular organisms). The multilevel-structure of the involved evolutionary processes is sketched schematically in Fig. 4.: the genome, i.e. the DNA sequence, codes not only for structural proteins, but also for a complex gene regulatory network (GRN). The dynamics of this GRN regulates the development of the phenotype. The environment influences development twofold, first by perturbations of the developmental process (noise), second by selection pressure for viable phenotypes. Organisms reproduce by duplication of their genome, 
which is an imperfect process frequently leading to errors (mutations). Typically, models of evolutionary adaptation focus either on sequence evolution or network structure alone, and hence imply a huge loss of information as compared to the true multi-level and multi-scale evolutionary dynamics. Artifical genomes could be an

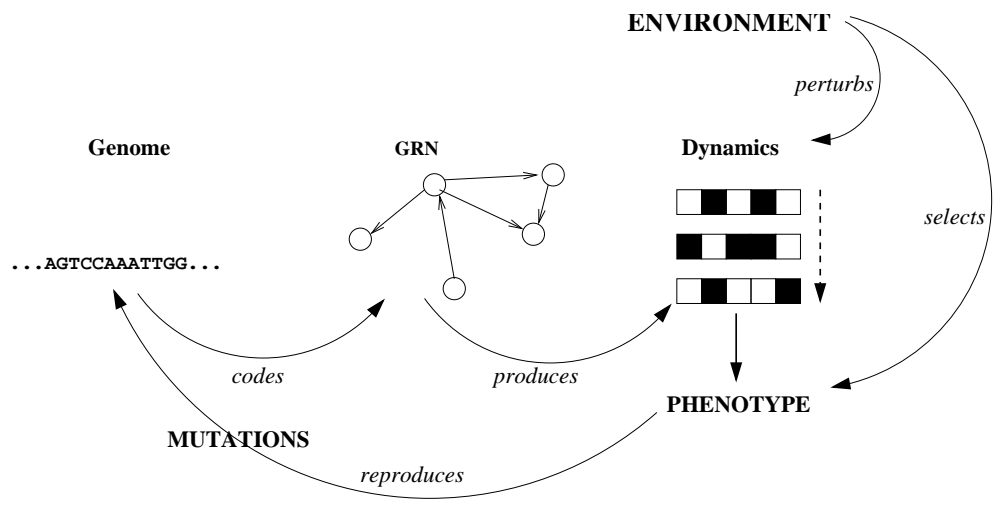

Fig. 4. Multilevel structure of the evolving genotype-phenotype map: besides coding for structural proteins, genomes also encode their own regulation by complex gene regulatory networks (GRN). GRN control the temporal and spatial dynamics that leads to "production" (development) of the organism (phenotype). The phenotype is reproduced by genome duplication, involving mutations. The environment can perturb developmental dynamics, as well as it selects for viable/adaptive phenotypes.

important step towards models that integrate these levels, and hence may lead to predictions on the effects of adaptive processes on sequence- and network evolution, and how these are related to each other.

\subsection{Definition of the evolutionary algorithm}

In this section, we briefly explore an example of an evolutionary scenario based on an artificial genome, motivated by the observation that development is highly canalized, i.e. buffered against both intrinsic and environmental noise, and mutations [19]. A number of studies has demonstrated that stabilizing selection for particular phenotypes leads to emergence of this high robustness, strongly facilitated by the high amount of neutrality contained in the fitness landscapes of complex regulatory networks [8]. Let us now define an evolutionary algorithm of stabilizing selection in a strongly fluctuating environment, based on an artificial genome. We start by generating an initial population of randomly assembled genomes; the number of bases $S$ is constrained such that each string contains exactly 64 genes. In all simulations discussed in the following, a promoter length $l_{p}=4$ and a gene length $l_{g}=6$ are applied. Next, different limit cycles of the associated RTNs are identified by running network dynamics, as defined in section 2.1 .1 , from $10^{4}$ dif- 
ferent random initial state configurations. This process is stopped when a RTN is identified which has a fixed point $S_{f}$ (a limit cycle of length one). In addition, we require that there can be identified at least 4 additional attractors, motivated from phenotype diversity frequently observed in many species [15]. Adaptation to unpredictable environments is often handled by stochastic switching between phenotypes and can lead to stabilization of even very small subpopulations of phenotypes that differ from the population majority [15], which we model by the requirement that the relative weight of the basin of attraction leading to $S_{f}$ should be small (less than $40 \%$ of the tested configurations). $S_{f}$ is the phenotype we want to stabilize, and the digit string $G_{f}$, that codes for its regulatory network, is the genotype we evolve.

We now apply stabilizing selection for $S_{f}$ as follows:

(1) Create a mutant $\bar{G}_{f}$ by random single base mutations, occurring with a probability $p_{m}=0.001$ per base.

(2) Run RTN dynamics from a random initial state, until an attractor is reached, otherwise stop after 200 iterations.

(3) If dynamics has converged to $S_{f}$, keep $\bar{G}_{f}$, otherwise keep $G_{f}$.

(4) For the next generation, iterate from (1).

We note that we disregard mutations of promoter sites, as well as mutation leading to new "genes", to avoid complications resulting from a varying genome size. Notice that, in step (2), we test only one initial configuration, corresponding to the fact that biological organisms are tested only against the environment they face at the current generation. Robustness against fluctuations, i.e. the capacity to stabilize the phenotype under diverse perturbations of development by unpredictable environmental influences as well as internal noise, is measured by running RTN dynamics for $G_{f}\left(\bar{G}_{f}\right)$ for a larger set $Z$ of initial configurations (e.g. $10^{4}$ random initial states). This variation in initial states simulates the fact that neither all a organisms in one generation meet a homogeneous environment, nor environments are constant over the course of generations. Then

$$
R_{f}(t):=\frac{Z_{f}(t)}{Z}
$$

defines the robustness against fluctuations, where $Z_{f}(t)$ is the fraction of initial states that lead to $S_{f}$ at generation $t$. A second measure of robustness is associated to the capacitance to buffer the system against disadvantageous mutations (mutational robustness $R_{m},[4]$ ). At each generation we measure the number of accepted mutants $P_{a}$ in the previous $P$ generations, and define

$$
R_{m}(t):=\frac{P_{a}(t)}{P} .
$$

If $P_{a}$, and hence $R_{m}$ increases with $t$, this indicates restructuring of the genome such that the probability of neutral or advantageous mutations with respect to $S_{f}$ has increased. 

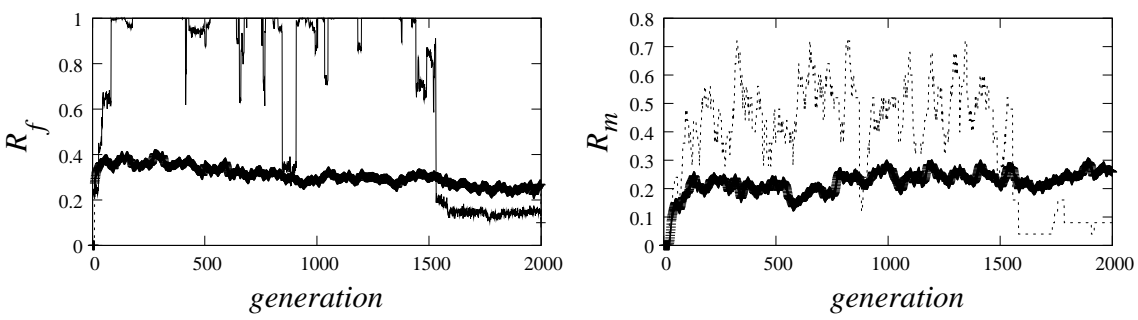

Fig. 5. Time course of evolutionary dynamics. Left: Evolution of the robustness $R_{f}$ against fluctuations in initial conditions, example of a particular run (thin-lined curve) and ensemble-average over 67 different evolutionary runs (thick-lined curve). Right: Evolution of the mutational robustness $R_{m}$, the dashed curve represents an example of an evolutionary run, the thick-lined curve the ensemble average.

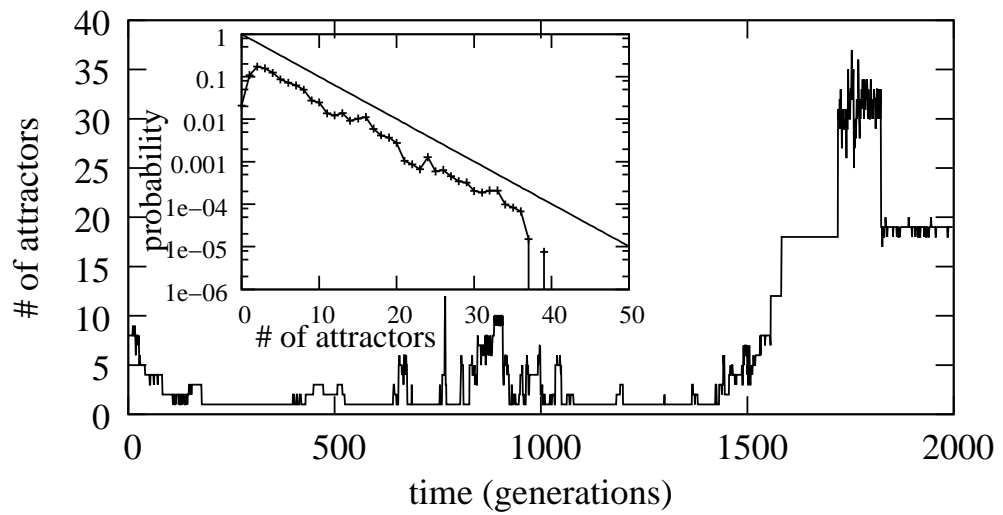

Fig. 6. Number of different dynamical attractors, as identified by sampling dynamics from $10^{4}$ random initial conditions in each generation, in a typical evolutionary run. Inset: statistical distribution of the number of indentified attractors, sampled from 67 evolutionary runs. The distribution exhibits an exponential decay (straight line shown for eye guidance). Notice that in about $2 \%$ of the cases, no attractors was identified due to the imposed length constraint on dynamical trajectories.

\subsection{Results}

Next, let us summarize the results obtained from evolutionary runs, starting from different random genome realizations with parameters as outlined in the previous paragraph.

\subsubsection{Evolution of robustness}

Figure 5 shows both quantities in a typical evolutionary run, and ensemble averages obtained from 67 evolutionary runs starting from different random genome realizations. Both $R_{f}$ and $R_{m}$ increase rapidly, however, exhibiting considerable 


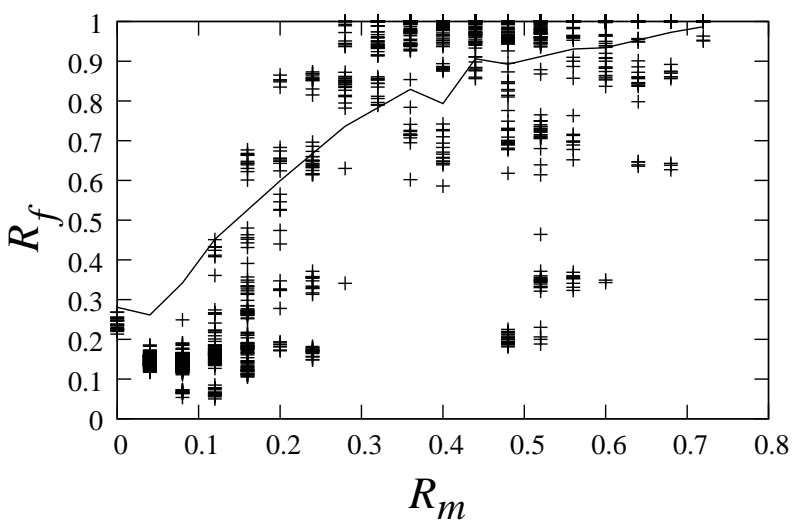

Fig. 7. Robustness $R_{f}$ against fluctuating dynamical initial conditions as a function of the robustness $R_{m}$ against point mutations of the genome sequence. Crosses show example results of a single evolutionary run, the lined curve is the ensemble average over 67 different runs. $R_{f}$ and $R_{m}$ exhibit a clear positive correlation.

fluctuations. In particular, $R_{f}$ exhibits an interesting intermittent dynamics reminiscent of a punctuated equilibrium [3], indicating metastability of the evolutionary dynamics. In fact, in most evolutionary runs we studied $R_{f}$ and $R_{m}$ could be stabilized only over a finite number of generations, as indicated in Fig. by the sharp decrease of both quantities around $t=1500$. The metastability is also visible in the ensemble average of $R_{f}$ (Fig. 5, left panel), which, after an initial sharp increase up to $R_{f} \approx 0.4$ shows a slight decline over the following generations. Another measure that can be applied to characterize the evolution of network dynamics is the number of different attractors (limit cycles) that are identified by the evolutionary algorithm in successive generations. Ideally, when stabilizing selection always succeeds, only the fixed point attractor corresponding to the phenotype $S_{f}$ should be present. Figure 6 shows that indeed most of the time the number of attractors is very small, however, there are intermittent increases (bursts). The inset of Fig. 6 shows that the statistical distribution of this quantity, as obtained from multiple evolutionary runs, exhibits an exponential decay. As it will be discussed later on, the "punctuated equilibrium" of evolutionary dynamics is both related to the selection criterion we chose, and to the mutation dynamics of the artificial genome, which is considerably different from single-link rewiring, as applied in most comparable "network only" studies.

$R_{m}$ is a measure of the probability that mutations are advantageous or neutral. In particular, neutrality of mutations strongly facilitates to find better phenotypes, since it allows evolution to explore a large number of different system configurations potentially leading to better phenotypes. When neutrality is a driving force of evolutionary dynamics, we expect that robustness against deleterious mutations and fitness of evolved phenotypes, i.e. $R_{m}$ and $R_{f}$, are correlated. As becomes evi- 
dent from Fig. [7, $R_{f}$ and $R_{m}$ are indeed positively correlated, similar to the results reported e.g. in [4].

The artificial genome now allows us to further investigate the effects of this evolutionary dynamics on both network and sequence structure.

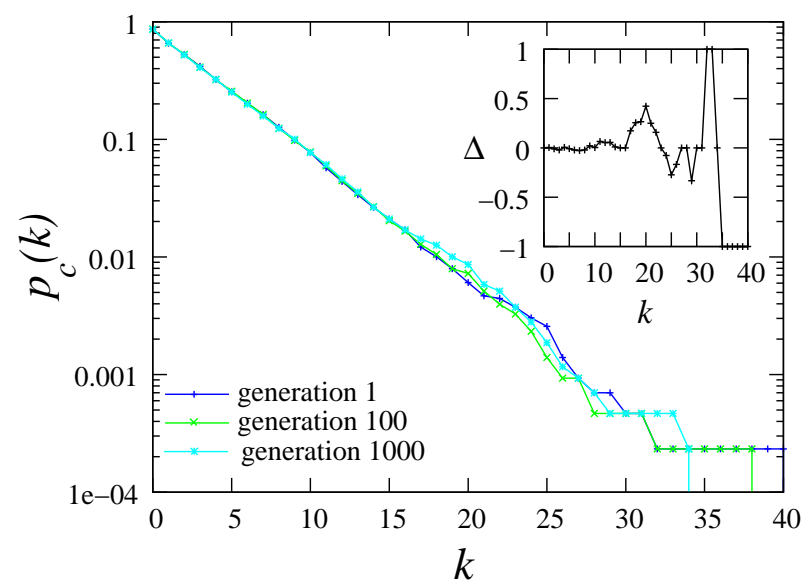

Fig. 8. Cumulative degree distributions $p_{c}(k)$ of incoming regulatory links (indegree distribution) for generation 1, 500 and 1000, averaged over 100 evolutionary runs. Inset: Relative difference $\Delta:=\left(p_{c}(t=1000)-p_{c}(t=1)\right) / p_{c}(t=1)$ between the distributions at generation 1 and 1000. Notice the increase around $k=18$, followed by a decrease for $k>20$ (details are discussed in the text).
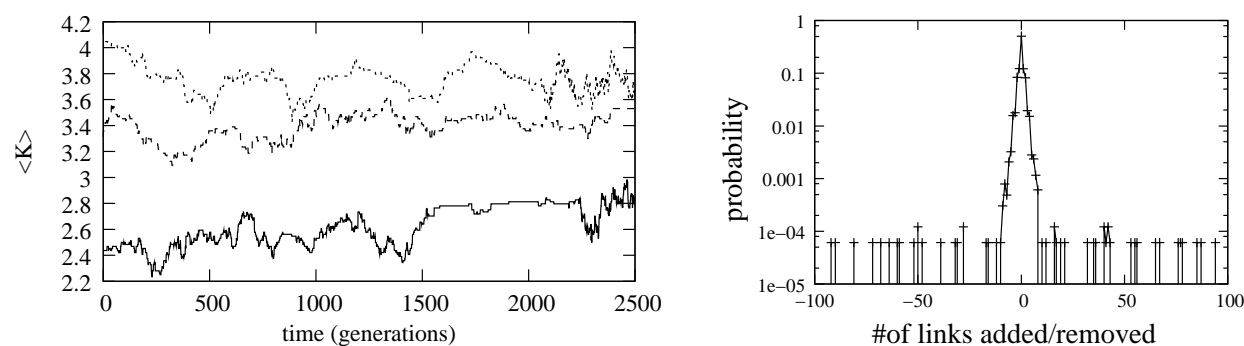

Fig. 9. Left panel: Evolution of the average network connectivity in three different runs. Right panel: statistical distribution of the number of links added/removed by point mutations in successive generations of evolving networks. Statistics was taken over all accepted mutants, averaged over 67 different evolutionary runs. About $50 \%$ of mutations are structurally neutral (no link added or removed), the rest shows a broad spectrum of rewiring effects at the network level. 


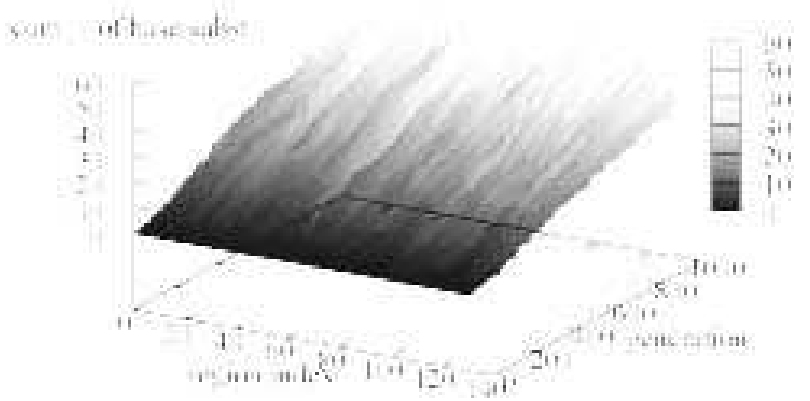

Fig. 10. Cumulative number of base exchanges during evolution for different positions on the genome, averaged over regions containing 100 bases each, during the course of evolution from generation 1 to 1000 in a particular evolutionary run. The brightness in grayscale indicates the number of bases exchanges. Increasing ruggedness of the surface points at divergent evolution of genomic regions accumulating base changes with rates that differ by orders of magnitude.

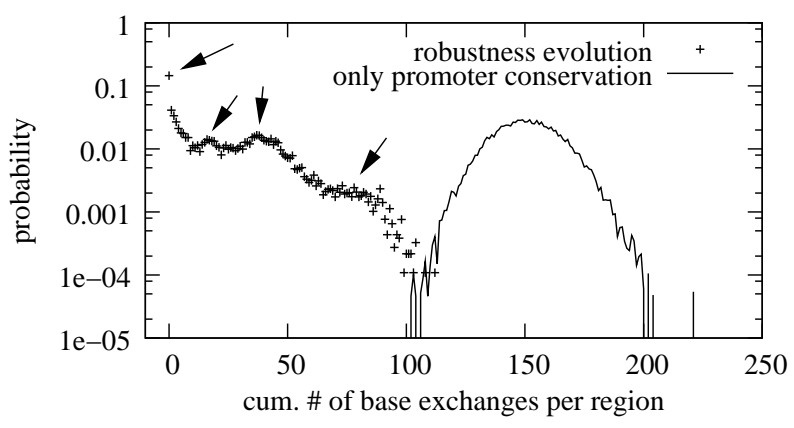

Fig. 11. Statistical distribution of the cumulative number of base exchanges per region at generation 2000, averaged over 100 different evolutionary runs. Left curve $(+)$ : evolution with the dynamical (robustness) constraint as described in the text. The distribution has a complex multimodal structure with maxima/plateaus around 0,40 and 80 (arrows). The lined curve on the right shows the same distribution for dynamically unconstrained evolution (keeping only promoter sequences fixed).

\subsubsection{Evolution of network- and sequence structure}

Let us first look on the evolution of network structure under the imposed dynamical robustness constraint. We performed 100 evolutionary runs with different initial assignments of $G_{f}$ and $S_{f}$; each simulation was observed over 2000 generations, and regulatory networks evolved after 2000 generations were compared to the initial networks. With regard to average network connectivity and outdegree distributions, no substantial reorganization was found. However, moderate reorganization is found in the distribution of regulatory inputs, which is shown in Fig. 8 (for smoothing of data, cumulative distributions $p_{c}(k):=$ probability to observe a node with indegree 
$\geq k$ are shown, besides averaging over 100 different evolutionary runs). Typically, an increase of probability for intermediate values of $k$ is found, while probability for larger $k$ is reduced. This can be clearly appreciated by investigation of the difference between both distributions (inset of Fig. 8). However, the overall shape of the distribution does not change significantly and still stays close to an exponential, presumably due to the small network sizes and limited number of generations observed. Figure 9 (left panel) shows the time evolution of the average network connectivity $\bar{K}$ for three different evolutionary runs. $\bar{K}$ shows considerable variance both with regard to different initial random genome realizations evolution starts from, as also with regard to fluctuations during evolution. In particular, the effects of point mutations at the sequence level on network wiring are strongly non-linear. To show this, we measured the statistical distribution of the number of regulatory links that were deleted or added in successive generations of accepted mutants, averaged over 100 different evolutionary runs (Fig. 9, right panel). In about 50\% of the cases, mutations did not affect network wiring; in the remaining cases, most often only one or a few links were affected (see the sharp peak around zero), however, there are also cases were a large number of links is added or removed simultaneously. This result is in contrast to many other studies of network evolution, which implicitly assume small, stepwise local changes in network wiring and hence only small moves along neutral paths of the fitness landscape. In our model, this is still the most frequent case, however, in some instances also larger jumps between different peaks of the fitness landscape naturally emerge through mutations in the sequence-based encoding of network structure that affect a large number of regulatory interactions.

Last, let us investigate how evolution proceeds at the most basic level of the system, i.e. the digit sequence of the artificial genome. Figure 10 shows the number of base exchanges during evolution for different positions on the genome. At each generation, the cumulative number of base substitutions in successive slices of 100 digits on the genome string, identified by a unique region index, during all previous generations was monitored. Increasing ruggedness of the surface points at divergent evolution of genomic regions accumulating base changes with at very different rates, giving evidence that there co-exist highly conserved and "adaptive" regions. We hypothesize that the former encode the invariant "core" of the regulatory network needed to produce the phenotype, while the latter contain neutral mutations, or support buffering against fluctuations. In Fig. 11, the statistical distributions for cumulative number of base substitutions in evolved genomes are compared to the control experiment without robustness constraint, only requiring preservation of promoter- and gene sequences. While in the control experiment, a simple, symmetric binomial distribution is found, evolved networks exhibit a strongly asymmetric, multi-modal distribution with at least three identifiable maxima/plateaus (indicated by arrows). This demonstrates that the selective constraint on regulatory dynamics indeed strongly influences the evolutionary patterns found in the genome at sequence level. 


\section{Discussion and Conclusions}

We studied statistical properties of the artificial genome model proposed by Reil [17] both on the level of base sequences and regulatory networks generated with this model, and the evolution of developmental canalization (robustness against noise in initial conditions of regulatory dynamics and against single base mutations). We find that random realizations of the artificial genome exhibit pronounced differences between the statistical distributions of regulatory inputs and outputs, and a scaling in the number of genes (as a function of the number of DNA bases) compatible with corresponding data of prokaryotic organisms for choices of model parameters as typically applied in our simulations. The simulation of evolutionary dynamics yields a number of surprising results. First, we observe that robustness is an evolvable property, and in particular that robustness against deleterious mutations and robustness against noise are correlated, similar to results of other studies [4]. However, while in most "network only" studies (without a sequence based description) only small adaptive changes (rewirings) are considered, we find emergence of highly nonlinear effects between sequence point mutations and network wiring (as predicted in [22] for random genome realizations), including a large number of structurally neutral mutations, and mutations that lead to simultaneous rewiring of multiple connections. This means that, while stepwise evolution along neutral paths of the fitness landscape with regard to phenotypic effects of mutations $[3,4,8]$ is still the main driving mechanism, also larger jumps between different peaks of the fitness landscape are possible. Interestingly, we found evidence that this increased nonlinearity in the genotype-phenotype map and the resulting fitness landscape tends to weaken the effectiveness of stabilizing selection in the long run, and the degree of evolved robustness exhibits considerable fluctuations during evolutionary runs. Concerning network structure, we found only moderate reorganization of the statistical distributions of input- and output numbers per node during evolution. In contrast, evolution leaves clearly visible signs at sequence level with a pronounced pattern of strongly conserved regions, and other parts of the genome evolving in a much less constrained way.

To conclude, the results of our study indicate that artificial genomes represent an interesting step towards more realistic models for the evolution of gene regulatory networks (GRN), by taking into account the indirect evolution of GRN structure through mutation of regulatory sequences, which cannot be accounted for in "network only" models. Clearly, the results of the current study are limited in scope; in future extensions of the model, we will in particular address variations in the number of genes (e.g. resulting from sequence duplications), and more realistic models for the binding of transcription factors to regulatory binding sites.

\section{References}

[1] Wolfgang Banzhaf. On the dynamics of an artificial regulatory network. In W. Banzhaf, T. Christaller, P. Dittrich, J. Kim, and J. Ziegler, editors, Advances 
in Artificial Life, Proceedings of the 7th European Conference (ECAL-2003), Dortmund, September 15-17, 2003, Lecture Notes in Artificial Intelligence, LNAI 2801, pages 217-227. Springer, Berlin, 2003.

[2] S. Bornholdt and T. Rohlf. Topological evolution of dynamical networks: Global criticality from local dynamics. Phys. Rev. Lett., 84:6114-6117, 2000.

[3] S. Bornholdt and K. Sneppen. Neutral mutations and punctuated equilibrium in evolving genetic networks. Phys. Rev. Lett., 81:236-239, 1998.

[4] Stefano Ciliberti, Oliver C. Martin, and Andreas Wagner. Robustness can evolve gradually in complex regulatory networks with varying topology. PLoS Computational Biology, 3:e15, 2007.

[5] Hidde de Jong. Modeling and simulation of genetic regulatory systems: A literature review. J. Comp. Biol., 9:67-103, 2002.

[6] Radu Dobrin, Quasim K. Beg, A. L. Barabási, and Z. N. Oltvai. Aggregation of topological motifs in the escherichia coli transcriptional regulatory network. $B M C$ Bioinformatics, 5:10, 2004.

[7] P. Erdhös and A. Rényi. On the evolution of random graphs. Publ. Math. Inst. Hung. Acad. Sci., 5:17-61, 1960.

[8] Pau Fernandez and Ricard Sole. Neutral fitness landscapes in signalling networks. J. R. Soc. Interface, 4:41-47, 2007.

[9] Leon Glass. The logical analysis of continous, non-linear biochemical control networks. J. Theor. Biol., 39:103-129, 1973.

[10] J. Hallinan and J. Wiles. Asynchronous dynamics of an artificial genetic regulatory network. In Ninth International Conference on the Simulation and Synthesis of Living Systems (ALife9), September 2004.

[11] I.K. Jordan, L. Mari-Ramez, Y.I. Wolf, and E.V. Koonin. Conservation and coevolution in the scale-free human gene coexpression network. Mol Biol Evol., 21:2058-2070, 2004.

[12] S.A. Kauffman. Metabolic stability and epigenesis in randomly connected nets. J. Theor. Biol., 22:437-469, 1969.

[13] Paul Dwight Kuo, Andre Leier, and Wolfgang Banzhaf. Evolving dynamics in an artificial regulatory network model. In Yao X., Burke E., Lozano J.A., Smith J., MereloGuerv J.J., Bullinaria J.A., Rowe J., Tino P., Kab A., and Schwefel H.-P., editors, Proc. of the Parallel Problem Solving from Nature Conference (PPSN-04), Birmingham, UK, September 2004, pages 571-580. Springer, LNCS 3242, Berlin, 2004.

[14] K.E. Kürten. Correspondence between neural threshold networks and kauffman boolean cellular automata. J. Phys. A, 21:L615-L619, 1988b.

[15] Edo Kussell and Stanislas Leibler. Phenotypic diversity, population growth, and information in fluctuating environments. Science, 309:275-278, 2005.

[16] A. Leier, D.P. Kuo, and W. Banzhaf. Analysis of preferential network motif generation in an artificial regulatory network model created by duplication and divergence. Advances in Complex Systems, 10:155 - 172, 2007.

[17] T. Reil. Dynamics of gene expression in an artificial genome - implications for biological and artificial ontogeny. In Proceedings of the 5th European Conference on Artificial Life, pages 457-466. Springer, 1999.

[18] T. Rohlf and S. Bornholdt. Criticality in random threshold networks: Annealed approximation and beyond. Physica A, 310:245-259, 2002.

[19] Mark L. Siegal and Aviv Bergman. Waddington's canalization revisited: Developmental stability and evolution. Proc. Natl. Acad. Sci., 99:10528-10532, 2002.

[20] D. Thieffry and D. Romero. The modularity of biological regulatory networks. BioSystems, 50:49-59, 1999. 
$\begin{array}{lllll}\text { September } & 18, & 2018 & 20: 23 & \text { WSPC/INSTRUCTION }\end{array}$

[21] A. Wagner. Robustness against mutations in genetic networks of yeast. Nature Genetics, 24:355-361, 2000.

[22] J. Watson, N. Geard, and J. Wiles. Towards more biological mutation operators in gene regulation studies. Biosystems, 76:239-248, 2004. 